\title{
Intraspecific Geographic Variation of Fragrances Acquired by Orchid Bees in Native and Introduced Populations
}

\author{
Santiago R. Ramírez • Thomas Eltz • Falko Fritzsch • \\ Robert Pemberton • Elizabeth G. Pringle • \\ Neil D. Tsutsui
}

Received: 15 March 2010/Revised: 31 May 2010 /Accepted: 16 June 2010 / Published online: 11 July 2010

(C) The Author(s) 2010. This article is published with open access at Springerlink.com

\begin{abstract}
Male orchid bees collect volatiles, from both floral and non-floral sources, that they expose as pheromone analogues (perfumes) during courtship display. The chemical profile of these perfumes, which includes terpenes and aromatic compounds, is both species-specific and divergent among closely related lineages. Thus, fragrance composition is thought to play an important role in prezygotic reproductive isolation in euglossine bees. However, because orchid bees acquire fragrances entirely from exogenous sources, the chemical composition of male perfumes is prone to variation due to environmental heterogeneity across habitats. We used Gas Chromatography/Mass Spectrometry (GC/MS) to characterize the
\end{abstract}

Electronic supplementary material The online version of this article (doi:10.1007/s10886-010-9821-3) contains supplementary material, which is available to authorized users.

S. R. Ramírez $(\bowtie) \cdot$ N. D. Tsutsui

Environmental Science, Policy \& Management,

University of California Berkeley,

137 Mulford Hall \#3114,

Berkeley, CA 94720, USA

e-mail: sramirez77@berkeley.edu

T. Eltz $\cdot$ F. Fritzsch

Department of Neurobiology,

Sensory Ecology Group, University of Düsseldorf,

Universitätstr. 1,

40225 Düsseldorf, Germany

\section{R. Pemberton}

Invasive Plant Research Laboratory,

United States Department of Agricultural Research Service,

3225 College Avenue,

Fort Lauderdale, FL 33314, USA

E. G. Pringle

Department of Biology, Stanford University,

Stanford, CA 94305, USA perfumes of 114 individuals of the green orchid bee (Euglossa aff. viridissima) sampled from five native populations in Mesoamerica and two naturalized populations in the southeastern United States. We recorded a total of 292 fragrance compounds from hind-leg extracts, and found that overall perfume composition was different for each population. We detected a pronounced chemical dissimilarity between native (Mesoamerica) and naturalized (U.S.) populations that was driven both by proportional differences of common compounds as well as the presence of a few chemicals unique to each population group. Despite these differences, our data also revealed remarkable qualitative consistency in the presence of several major fragrance compounds across distant populations from dissimilar habitats. In addition, we demonstrate that naturalized bees are attracted to and collect large quantities of triclopyr 2butoxyethyl ester, the active ingredient of several commercially available herbicides. By comparing incidence values and consistency indices across populations, we identify putative functional compounds that may play an important role in courtship signaling in this species of orchid bee.

Key Words Hymenoptera · Monoterpenes . Sesquiterpenes · Pheromone evolution · Euglossini . Invasive species $\cdot$ Orchids $\cdot$ Bees $\cdot$ Triclopyr BEE

\section{Introduction}

Most insects rely on chemical compounds (pheromones) to identify and attract conspecific mates (Wyatt, 2003). The chemical composition of mating pheromones may range from single molecules to diverse, complex mixtures (Symonds and Elgar, 2004, 2008; Billeter et al., 2009). Because mating pheromones usually consist of species- 
specific blends, they often mediate prezygotic reproductive isolation among closely related lineages (Cardé et al., 1977; Roelofs et al., 2002). In insects, mating pheromones are synthesized de novo or from sequestered precursors. In either case, both biotic and abiotic factors influence pheromonal chemical composition (Stennett and Etges, 1997; Groot et al., 2009). Hence, an important question is how insect populations that colonize novel environments respond to environmental changes that influence mating pheromones. In addition, to understand the underlying mechanisms of pheromone evolution in insects, we must examine intraspecific phenotypic variation in pheromone chemistry across habitats, geographic regions, and genetic gradients.

Orchid (or euglossine) bees constitute a diverse lineage of Neotropical insect pollinators (Ramírez, 2009; Ramírez et al., 2010) that are best known for their fragrancecollecting behavior (Darwin, 1862; Dressler, 1968; Dodson et al., 1969). Male bees collect volatile compounds from multiple floral and non-floral sources that they use subsequently during courtship display (Dressler, 1982; Eltz et al., 2005b). Males of all species of orchid bees ( 200) have enlarged hind-tibial organs in which they deposit perfumes that consist primarily of terpenoids and aromatic compounds (Williams and Whitten, 1983; Eltz et al., 1999; Zimmermann et al., 2009a). Male orchid bees land on the surface of fragrance sources and secrete large quantities of long-chain lipids from cephalic labial glands that dissolve the volatile compounds to be collected (Whitten et al., 1989; Eltz et al., 2007). Although it has not been confirmed directly via behavioral experiments, it is likely that perfume chemistry enables females to choose mates and/or discriminate non-specific males (Eltz et al., 1999, 2003, 2005a; Bembé, 2004; Zimmermann et al., 2006). In a recent study, Zimmermann et al. (2009a) analyzed the chemical composition of the fragrances acquired by male orchid bees from 15 closely related sympatric species in the genus Euglossa from central Panama. They showed that related lineages of bees accumulate species-specific fragrance blends that have differentiated rapidly during lineage diversification, potentially in response to strong selection against hybridization or mating interference between species. Previous studies also have shown that perfume variation tends to be lower within lineages than between lineages (Eltz et al., 1999, 2005a, 2008; Zimmermann et al., 2006), even when individuals co-occur in the same habitat (Eltz et al., 2005a, 2008). However, the extent of intraspecific perfume variation across geographic regions and genetic gradients has not yet been examined.

Male orchid bees may accidentally accumulate a considerable diversity of inactive (noisy) compounds during the process of perfume collection (Eltz et al., 2005a), as they often collect volatiles from multiple sources, even at local scales (Pemberton and Wheeler, 2006). In addition, variation in the availability and abundance of specific chemicals among habitats may result in disparate fragrance phenotypes (Eltz et al., 2005a). Thus, orchid bees must deal with environmentally induced variability while maintaining a reliable chemical communication system. What proportion of fragrance compounds is biologically active, and thus required for mating? Do conspecific male orchid bees collect similar compounds across disparate habitats? Can different chemical compounds serve homologous functions in distant populations? We designed a comprehensive analysis of fragrance variation within and between interbreeding populations of a single species, Euglossa aff. viridissima, to answer some of these questions. In particular, we focused on elucidating phenotypic change in the perfumes of $E$. aff. viridissima upon the invasion of a novel environment.

The green orchid bee Euglossa viridissima is distributed throughout lowland dry forests in northern Mesoamerica (Hinojosa-Diaz et al., 2009), ranging in distribution from southern Costa Rica to Northern Mexico (Fig. 1). Previous taxonomic treatments recognized E. viridissima as a single distinct species (e.g., Roubik and Hanson, 2004). However, a recent study of populations from the Yucatan peninsula (Mexico) showed instead that this lineage consists of two cryptic sister species (Eltz et al., 2008). The most prominent morphological difference between the two lineages is in the number of teeth on the mandible of males: two or three dentitions. Additionally, whereas males with three dentitions (hereafter $E$. aff. viridissima) collect large quantities of the compound 2-hydroxy-6-nona-1,3-dienyl-benzaldehyde (abbreviated HNDB), males with two dentitions (E. viridissima) lack HNDB in their perfumes (Eltz et al., 2008). A detailed systematic analysis of these lineages based on morphology, chemistry, and genetic data is in preparation (Eltz et al., unpublished).

As early as 2003 , bees of E. aff. viridissima were introduced accidentally and naturalized in southeastern United States (Skov and Wiley, 2005). Since then, vigorous populations have become established throughout most of the southern coastal peninsula of Florida (Pemberton and Liu, 2008; Liu and Pemberton, 2009; Pemberton and Ramírez, personal observation). A chemical analysis of the perfumes of naturalized bees from Florida showed that male bees collect multiple fragrance compounds from several plant species, including native, naturalized, and horticultural plants (Pemberton and Wheeler, 2006). However, the composition of these fragrances has not been compared with that of native $E$. aff. viridissima populations.

In the present study, we analyzed the chemical composition of the fragrances acquired by $E$. aff. viridissima across its native range in Mesoamerica and its introduced range in Florida (U.S.). We estimated the magnitude of 
Fig. 1 Map of Mesoamerica and southern United States indicating sampling localities where male Euglossa aff. viridissima were collected. Native range populations in Mesoamerica were El Chote, Monte Pio, El Remate, Xmatkuil and ACG. Naturalized populations in the United States were Gumbo-Limbo and Fern Forest

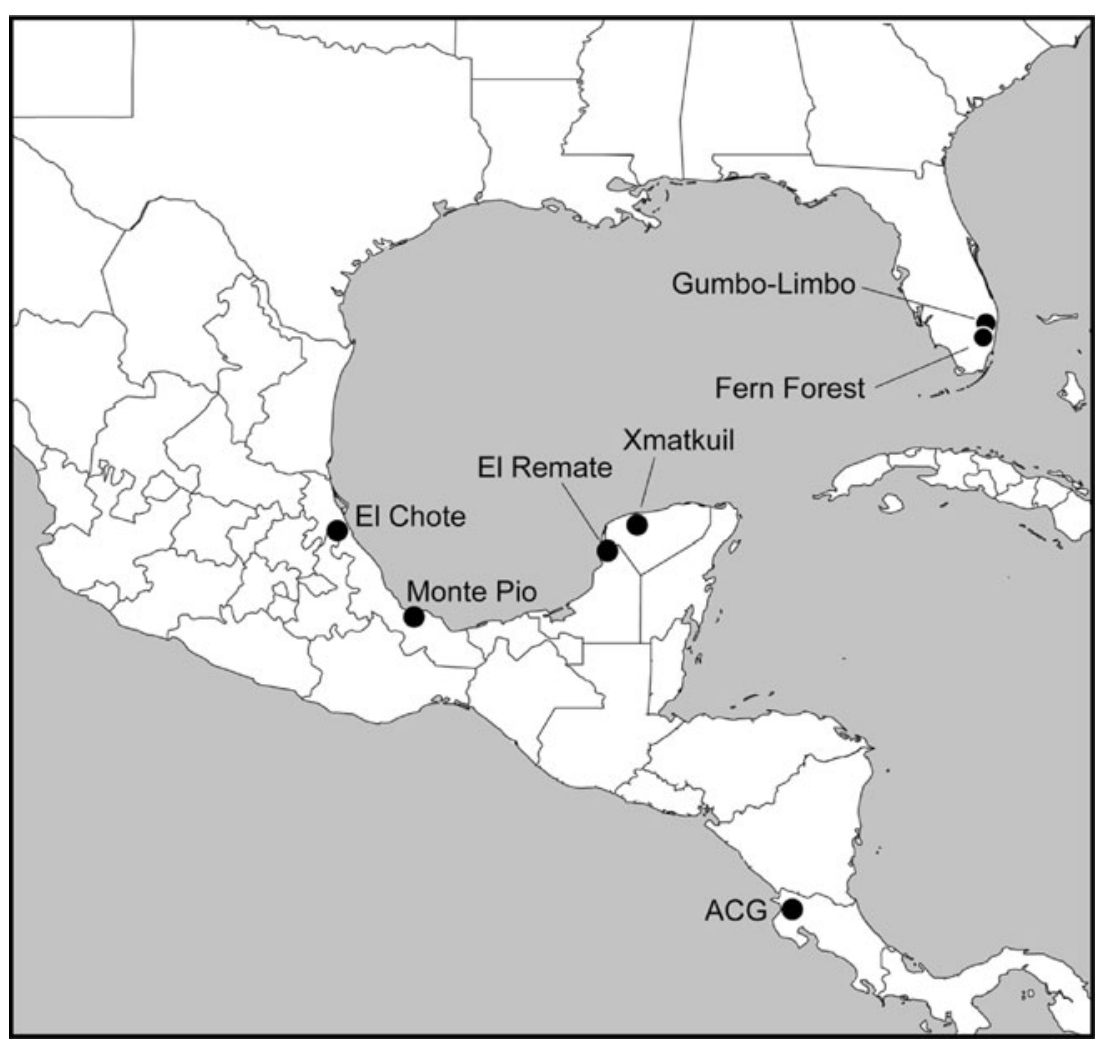

intraspecific geographical variation in fragrance composition and identified volatile compounds that vary within and between bee populations. In addition, because the recent introduction of E. aff. viridissima to the United States may have resulted in both a genetic bottleneck and a habitat shift, we asked whether changes in perfume chemistry have accompanied the naturalization of this lineage of bees.

\section{Methods and Materials}

Sampling A total of 114 male orchid bees belonging to the Euglossa aff. viridissima lineage were collected from five localities across the native range in Costa Rica and Mexico, and two localities in the introduced range in the U.S. (Florida) in 2008 and 2009 (Fig. 1; Supplementary Material Appendix 1). Male bees were lured with synthetic chemical baits consisting of squares of blotter paper impregnated with $p$-dimethoxybenzene, methyl cinnamate, or eugenol (Sigma-Aldrich, St. Louis, MO, USA; Eltz et al., 2008). The impregnated paper squares were suspended $1.5 \mathrm{~m}$ aboveground and covered with screen mesh to prevent male bees from accessing the bait. Baiting stations were monitored every 15 min from 9AM until 1PM. Males were captured with hand nets and kept in the shade inside screen cages until subsequent dissection, typically conducted the same day.
Florida populations were sampled at two natural area preserves. Fern Forest (Broward County) is primarily a tropical hardwood forest grading into freshwater cypress swamp and upland pine habitats. Gumbo-Limbo Nature Center (Palm Beach County) is located on a barrier island with a tropical hardwood forest and mangroves. Mesoamerican populations were sampled from various habitats, including secondary seasonally dry tropical forest (ACG), secondary seasonally dry scrubland (Xmatkuil), farmland surrounded by stretches of tropical perennial forest (El Chote), settlement adjacent to tropical perennial forest (Monte Pio), and coastal mangroves (El Remate).

Fragrance Extraction and Chemical Analysis Male bees were cold-anaesthetized either on ice or inside a freezer $\left(-20^{\circ} \mathrm{C}\right)$ for $5 \mathrm{~min}$ immediately prior to dissection. Right hind tibiae were removed with clean dissecting scissors and deposited in $2 \mathrm{ml}$ screw-cap autosampler vials (Agilent Technologies, Santa Clara, CA, USA), which contained $500 \mu \mathrm{l}$ of hexane to extract the fragrances. To distinguish between exogenous volatiles and endogenous maleproduced lipids, acetates, and straight-chain hydrocarbon compounds, labial glands were dissected, extracted, and analyzed from a subset of individuals $(N=43)$ using the same protocol described above. All compounds present in both leg extracts and labial glands were excluded from the analysis (Fig. 2). Extracted samples were stored at $-20^{\circ} \mathrm{C}$ 
Fig. 2 Overlaid total ion current chromatograms corresponding to hind-leg (black) and labial gland (grey) extracts from the same individual bee. Compounds present in both hind legs and labial glands were considered endogenous in origin and thus were excluded from the analysis. The $\mathrm{Y}$ axis indicates ion abundance

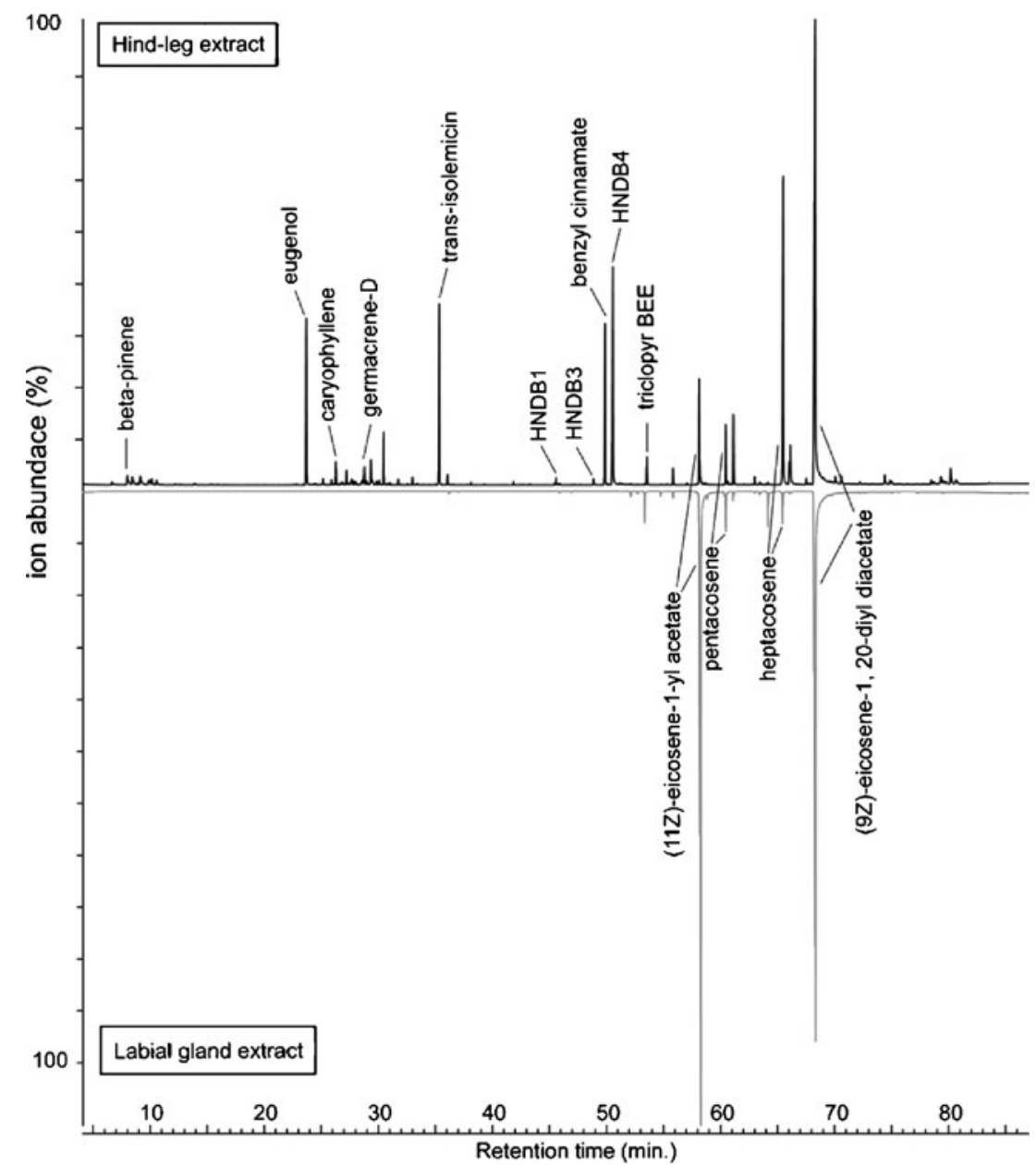

until subsequent GC/MS analysis. Voucher specimens of all samples were either pinned or deposited in 200 proof ethanol.

Gas Chromatography/Mass Spectrometry (GC/MS) was conducted at the Department of Environmental Sciences, Policy and Management at the University of California Berkeley, using a 7890A Agilent GC coupled with a 5975C Agilent Mass Selective Detector (Agilent Technologies, Santa Clara, CA, USA). The GC was fitted with a $30 \mathrm{~m}$ long, $0.25 \mathrm{~mm}$ internal diam, non-polar Agilent HP-5MS capillary column (cat. \# 19091S-433). Sample aliquots of $1 \mu \mathrm{l}$ were injected by using an Agilent 7683B automatic injector operated in splitless mode. The oven temperature was programmed from 60 to $300^{\circ} \mathrm{C}$ at $3^{\circ} \mathrm{C} / \mathrm{min}$, using helium as carrier gas with a constant flow rate set to $0.7 \mathrm{ml} /$ min. Mass Selective Detector (MSD) scanning parameters ranged from 50 to $550 \mathrm{amu}$, with a sampling rate of 2.91 scans/sec and a threshold detection of 150 counts.

Chemical analyses revealed the presence of triclopyr 2butoxyethyl ester (hereafter triclopyr BEE), in Florida populations only. Triclopyr BEE is the active ingredient of a variety of commercial herbicides. To determine whether male E. aff. viridissima were actively attracted to this compound, we performed attraction bioassays with both captive and wild bees. Captive bees were nest-trapped in Florida (August 2009) and reared at UC Berkeley in an insectary facility maintained at $25^{\circ} \mathrm{C}, 40-60 \%$ humidity, and kept under a 12/12 h light/dark cycle. Male bees were not exposed to any fragrances prior to the bioassay, in which we presented male bees with hind leg extracts that contained large quantities of triclopyr BEE and as little as possible of other volatile compounds. We applied $100 \mu \mathrm{l}$ of the hexane extract to clean blotter paper squares placed inside a flight cage where the bees were reared. As an experimental control, we presented male bees with labial gland extracts from the same individuals for which fragrance extracts were selected in the experimental treatment. The number of bees displaying fragrance gathering behavior within the first 2 min was recorded.

In addition, to test whether male $E$. aff. viridissima from native-range populations also were attracted to triclopyr BEE, we purified this compound from leg extracts using a preparative GC system similar to that of Nojima et al. (2008). A megabore DB-5 capillary column $(30 \mathrm{~m}$, 
$0.53 \mathrm{~mm}$ ID, $0.5 \mathrm{um}$ film thickness) was used to separate compounds in an HP 5890 II gas chromatograph (50 to $300^{\circ} \mathrm{C}$ at $\left.10^{\circ} / \mathrm{min}\right)$. Injection was on column, with a $30 \mathrm{~m}$ retention gap preceding the actual analytical column. The peak of triclopyr BEE was captured by using a $40 \mathrm{~cm}$ piece column trap (HP-1, 0.53 ID) that was connected to the preparative outlet at the time of elution. The substance retained in the trap was washed out with $n$-hexane. Repeated runs yielded approximately $75 \mu \mathrm{g}$ of purified triclopyr BEE diluted in $1 \mathrm{ml}$ of $n$-hexane. Subsequently, we presented male $E$. aff. viridissima with purified extracts of triclopyr BEE. The bioassay was conducted in a disturbed dry forest near Xmatkuil (Merida, Mexico) during the morning of 14 March, 2010. We applied $200 \mu \mathrm{l}$ of isolated triclopyr BEE in $n$-hexane $(\sim 15 \mu \mathrm{g})$ to filter paper (Whatman 1,2 cm) pinned to a small tree at breast height. In the vicinity ( $>4 \mathrm{~m}$ away), we also exposed two $p$ dimethoxybenzene baits, which were visited by dozens of male $E$. aff. viridissima during the same morning. Bees that landed on the triclopyr BEE filter paper were observed briefly to verify volatile collection.

Compound Characterization We used the software Chemstation vE.02.00 (Agilent Technologies) to register chromatogram peaks and save their corresponding spectra in user-built mass-spectral libraries (Ramírez and Eltz, unpublished) which we use to cross-reference additional chromatograms. Libraries were recursively updated as new compounds were found. We further characterized individual compounds by comparing spectra against several published libraries (Adams, 2001; pal600K, Palisade Corporation, USA). Authentic standards of monoterpene and sesquiterpene compounds (Sigma-Aldrich, St. Louis, MO, USA), injected and analyzed under the same conditions and instrument described above (UC Berkeley), were also used to corroborate compound identities (Table 1). Uncharacterized compounds were named based on retention times and EI-mass spectrum ions (Table 1). Automatic peak integration was conducted using the RTE integrator in the software Chemstation vE.02.00 (Agilent Technologies) set to a minimum-area detection threshold equivalent to $0.5 \%$ of the area of the largest peak.

Statistical Methods We built a square matrix containing the absolute quantities (total ion currents) for each compound, containing all samples from all localities. Total ion currents were transformed to relative amounts (percentage) per individual. We compared individual chemical profiles within and between populations via non-metric Multidimensional Scaling (MDS), an ordination technique where a predetermined number of axes of variation are chosen, and non-metric distances are fitted to those dimensions. Because ordination via MDS makes few assumptions about the nature of the data, any distance measure can be applied. We calculated a triangular distance matrix between samples (individuals) using the Bray-Curtis index of dissimilarity, which has the advantage of being insensitive to compounds jointly absent in sample pairs (i.e., pairwise dissimilarities are fixed). We computed 2- and 3-dimensional MDS plots (50 iterations per run) using the software package ecodist v1.2.2 (written in $\mathrm{R}$ ). We ran each analysis 10 times; convergence between solutions was visually inspected. To assess statistically whether orchid bee fragrances exhibit greater dissimilarity between populations than within populations, we conducted an Analysis of Similarity (ANOSIM) test, implemented in the software package vegan v1.15-4 (written in R). Additional descriptive statistics, tests, and plots were produced using $\mathrm{R}$ basic packages, freely available at www.cran.r-project.org. We estimated the relative contribution of individual compounds to the observed ordinal dissimilarities using the Similarity Percentage (SIMPER) method, implemented in the software package primer v6 (Clarke and Gorley, 2006). All MDS calculations were verified using the software primer.

\section{Results}

We registered a total of 333 compounds in hind leg extracts of 114 males sampled from seven populations (Fig. 1). By comparing the chemical profiles of hind leg extracts against those from labial glands (Fig. 2) we determined that 41 compounds were produced in the bees' labial glands (endogenous origin), and included straight chain hydrocarbons, acetates, diacetates, and alcohols (Supplementary Material Appendix 2). The remaining 292 compounds are thus exogenous in origin, and included monoterpenes, sequiterpenes, bicyclic sesquiterpenes, triterpenes, and other compounds (Table 1). The per-capita number of exogenous compounds across all populations ranged from 1 to 65 chemicals (average $21.50 \pm 12.66$ ) with marginally similar means in all seven populations (ANOVA $F=3.2118$, $P=0.075$ ). On average, male bees from Fern Forest (U.S.) exhibited the lowest number of compounds per capita (15.83 \pm 10.08$)$, whereas El Remate (Mexico) exhibited the highest number $(29.23 \pm 6.24$; Fig. 3$)$. When the two U.S. populations were combined, they had significantly fewer compounds per individual $(17.46 \pm 10.78)$ than all Mesoamerican populations combined (24.14 \pm 13.17 ; ANOVA $F=8.0419, P=0.005$ ).

Our non-metric Multidimensional Scaling (MDS) analysis included exogenous compounds only. When all volatile compounds in the data matrix were included (coded by relative abundances), a strong clustering by population was found (Fig. 4a). This observation was corroborated by the 
Table 1 Most common exogenous fragrance compounds collected by male Euglossa aff. viridissima ranked by their incidence (\%) across populations

Compound name

Compound class Retention Entry Incidence Contribution time \# (\%) to dissimilarity (min) $\quad(\%)$ USA vs. Mesoamerica $^{a}$

eugenol

2-hydroxy-6-nona-1,3-dienyl-benzaldehyde 4 (HNDB4)

caryophyllene

isolemecin, trans-

ocimene, beta-

benzyl benzoate

2-hydroxy-6-nona-1,3-dienyl-benzaldehyde 1 (HNDB1)

m/z:55,69,81,95,107,119,135,147,161,175,189,207,218,426

2-hydroxy-6-nona-1,3-dienyl-benzaldehyde 3 (HNDB3)

$\mathrm{m} / \mathrm{z}: 53,65,74,92,120,155$

humulene, alpha-

cadinene, delta-

benzyl cinnamate

m/z:55,68,81,93,107,121,133,147,161,175,189,204

pinene, alpha-

m/z:55,69,81,95,107,119,135,147,161,175,189,203,218

3-cyclohexane-1ol, 4-methyl-1m ethylethyl

2-hydroxy-6-nona-1,3-dienyl-benzaldehyde 2 (HNDB2)

triclopyr 2-butoxyethyl ester

germacrene, $\mathrm{D}$

similar to elemicin

similar to amorphene, alpha-

similar to elemicin

similar to amyrin, alpha-

1,4-dimethoxybenzene,

cubene, beta-

epizonarene

m/z:55,115,127,173,183,201,215,228,244,269,283,293,311,326,344

cineole, $1,8-$

copaene, alpha-

methyl ester

myrcene, beta-

bisabolene, beta-

pinene, beta-

barbatene, beta-

$\mathrm{m} / \mathrm{z}: 55,67,77,81,91,105,119,133,161,204$

$\mathrm{m} / \mathrm{z}: 65,77,91,115,129,147,175,244,260$

$\mathrm{m} / \mathrm{z}: 55,71,99,115,128,141,157,171,186,200,213,228,245,301,326$

$\mathrm{m} / \mathrm{z}: 55,69,83,95,108,115,127,145,159,173,183,201,215,225,244,253,285,299,324,342$

similar to amorphene, alpha-

\begin{tabular}{|c|c|c|c|c|}
\hline phenylpropanoid & 23.679 & 164 & 79 & 8.54 \\
\hline aromate & 50.698 & 54 & 74 & 19.04 \\
\hline sesquiterpene & 26.288 & 173 & 68 & 2.60 \\
\hline NA & 35.388 & 61 & 50 & 2.25 \\
\hline monoterpene & 10.547 & 199 & 44 & 3.44 \\
\hline aromate & 39.306 & 218 & 42 & 3.00 \\
\hline aromate & 45.628 & 51 & 41 & 1.65 \\
\hline triterpene & 80.675 & 59 & 40 & 1.25 \\
\hline aromate & 48.890 & 53 & 38 & 2.27 \\
\hline unknown & 16.572 & 373 & 36 & 1.17 \\
\hline sesquiterpene & 27.673 & 165 & 36 & 1.22 \\
\hline sesquiterpene & 30.465 & 110 & 35 & 0.07 \\
\hline NA & 49.863 & 208 & 34 & 3.48 \\
\hline sesquiterpene & 25.149 & 245 & 32 & 1.26 \\
\hline monoterpene & 6.639 & 174 & 28 & 0.61 \\
\hline unknown & 79.337 & 127 & 26 & 0.25 \\
\hline monoterpene & 15.926 & 180 & 25 & 0.23 \\
\hline aromate & 47.231 & 52 & 25 & 1.18 \\
\hline aromate & 53.553 & 76 & 25 & 17.37 \\
\hline sesquiterpene & 28.794 & 359 & 25 & 0.69 \\
\hline aromate & 31.781 & 413 & 25 & 0.48 \\
\hline sesquiterpene & 30.105 & 45 & 23 & 0.29 \\
\hline unknown & 31.753 & 49 & 23 & 0.33 \\
\hline triterpene & 79.800 & 424 & 23 & 0.55 \\
\hline aromate & 15.308 & 179 & 19 & 0.14 \\
\hline sesquiterpene & 28.789 & 194 & 19 & 1.03 \\
\hline sesquiterpene & 29.504 & 43 & 18 & 0.45 \\
\hline unknown & 64.722 & 333 & 18 & 0.28 \\
\hline monoterpene & 9.906 & 255 & 18 & 0.23 \\
\hline sesquiterpene & 24.486 & 182 & 18 & 0.20 \\
\hline NA & 36.067 & 235 & 18 & 1.79 \\
\hline monoterpene & 8.424 & 254 & 17 & 0.25 \\
\hline sesquiterpene & 29.922 & 378 & 16 & 0.82 \\
\hline monoterpene & 7.989 & 175 & 15 & 0.30 \\
\hline sesquiterpene & 27.227 & 188 & 15 & 0.17 \\
\hline sesquiterpene & 28.068 & 193 & 15 & 0.13 \\
\hline unknown & 51.766 & 72 & 15 & 0.26 \\
\hline unknown & 62.691 & 311 & 15 & 0.10 \\
\hline unknown & 66.279 & 334 & 15 & 0.16 \\
\hline sesquiterpene & 28.611 & 161 & 14 & 0.15 \\
\hline
\end{tabular}

${ }^{\text {a }}$ Similarity Percentage (SIMPER) was used to calculate the relative contribution of each compound to the observed dissimilarity between US and Mesoamerican populations. Values $>1 \%$ are indicated in bold

ANOSIM results, which indicated that fragrance dissimilarity within populations was lower than among populations $(R=0.433, P<0.001)$. We found a similar pattern when we excluded the two compounds with the highest contribution to the dissimilarity between U.S. and Mesoamerica (Fig. 4b; see SIMPER analysis below), namely HNDB4 (one of four stereoisomers) and triclopyr BEE. Likewise, MDS ordinations based only on the 50 compounds with the 


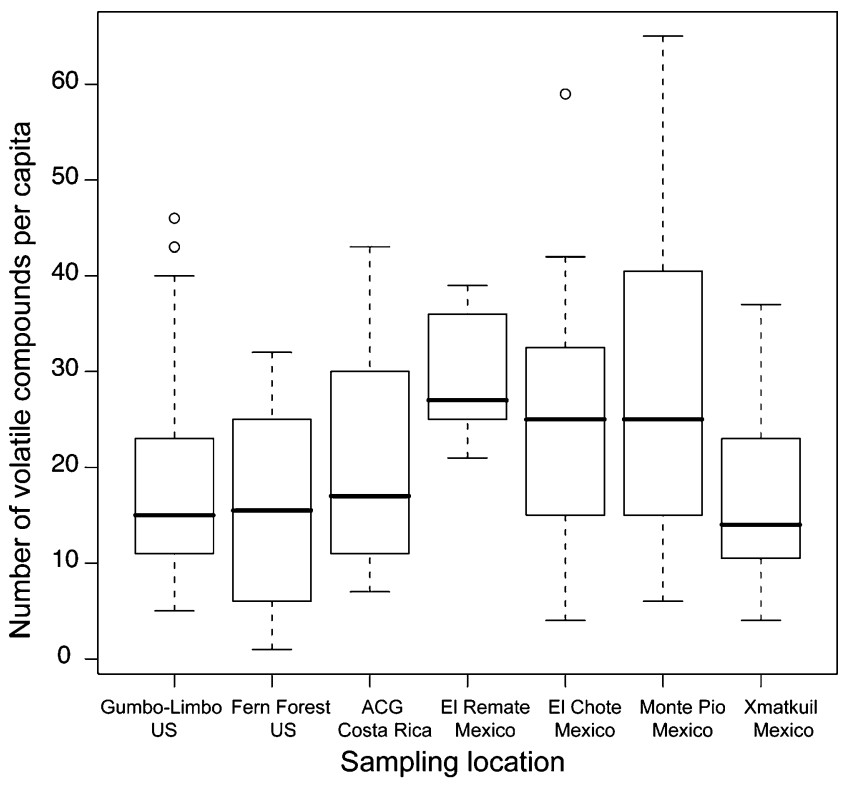

Fig. 3 Boxplots of the number of volatile exogenous compounds per capita in fragrances of male Euglossa aff. viridissima in native and introduced populations highest incidence ranks across all populations (Fig. 4c), and the entire dataset transformed into discrete binary (presence/absence) characters (Fig. 4d), also supported a strong differentiation between all populations. In addition, regardless of which data partition we used, we found that introduced (U.S.) and native (Mesoamerica) populations were strongly differentiated (Fig. 4; ANOSIM populations not pooled: $R=0.483, P<0.001)$. To explore the possibility that per-capita compound diversity (number of compounds) affected population differences, we overlaid a bubble-plot on an MDS ordination (based on all fragrance data) with circle diameters proportional to the number of compounds per capita (Fig. 5). Although mean per capita fragrance richness was marginally different among populations (Fig. 3), populations did not appear to cluster based on fragrance diversity alone (Fig. 5).

Our SIMPER analysis revealed that five compounds jointly contributed to $>50 \%$ of the observed chemical dissimilarity between populations from U.S. and Mesoamerica (Table 1). These included HNDB4 (19.04\%), triclopyr BEE (17.37\%), eugenol (8.54\%), benzyl cinnamate $(3.48 \%)$, and beta-ocimene $(3.41 \%)$. With the exception of triclopyr BEE, which was present only in U.S.
Fig. 4 Non-metric multidimensional scaling (nMDS) plots based on the chemical composition of exogenous compounds present in hind legs of male Euglossa aff. viridissima. Ordination plots were computed based on a all volatile compounds, b all volatile compounds except the two most dissimilar between U.S. and Mesoamerican populations (i.e., HNDB4, triclopyr BEE), c the 50 compounds with highest incidence across all populations, and $\mathbf{d}$ all volatile compounds coded as binary characters (presence/absence). Filled circles and triangles correspond to individuals from naturalized populations
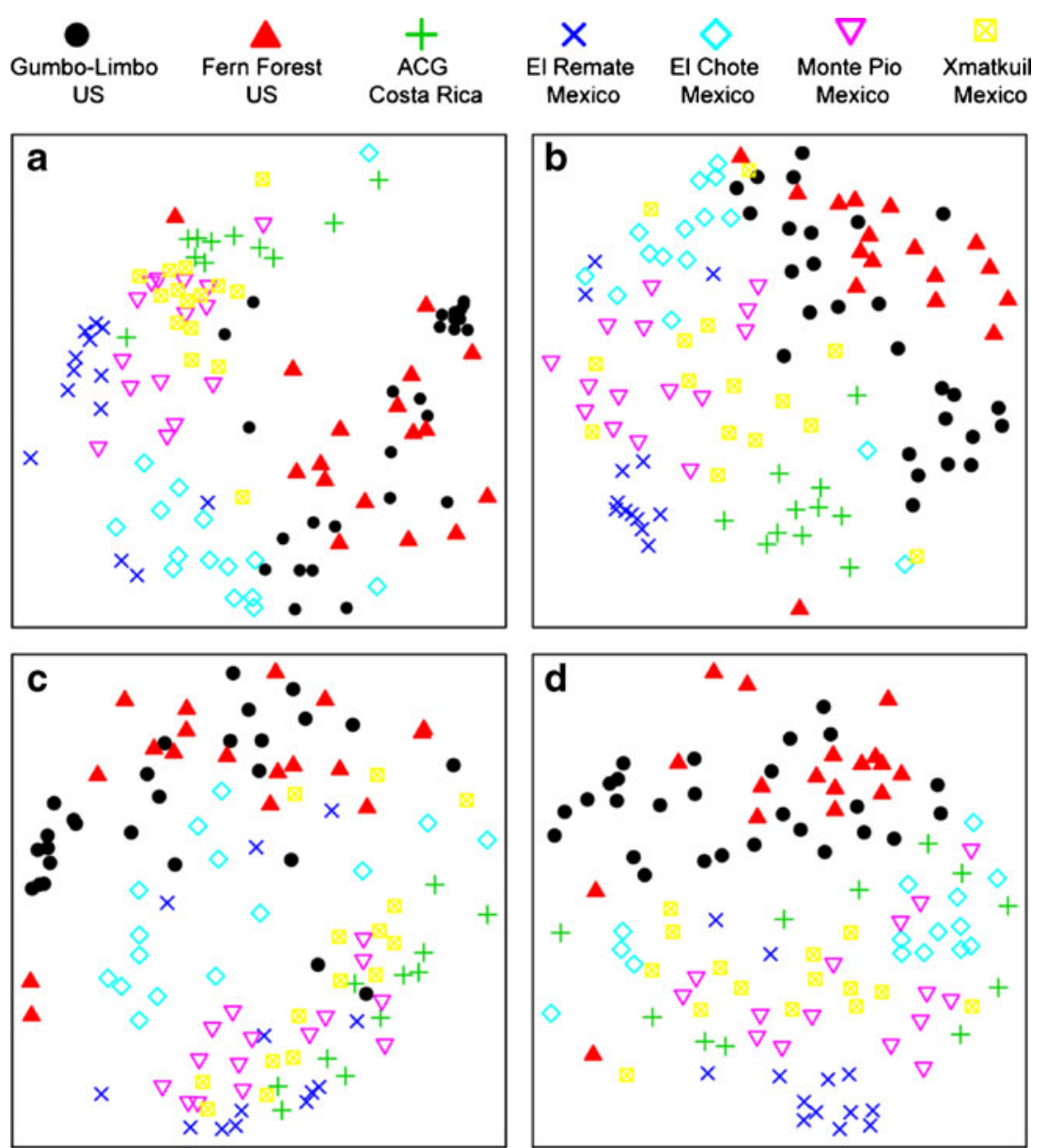
Fig. 5 Bubble plot overlaid on an MDS ordination based on all exogenous fragrance compounds. Circle diameters correspond to the number of volatile exogenous compounds present in the fragrances of each individual. Analyses were performed using the software primer (Clarke and Gorley 2006). Filled circles and triangles correspond to individuals from naturalized populations

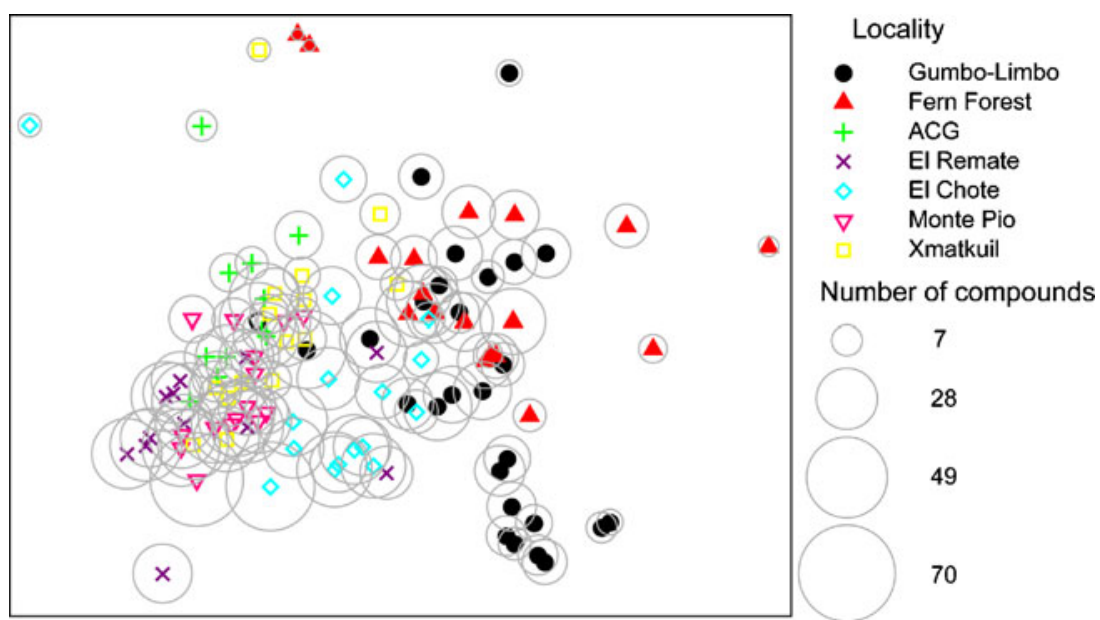

populations, these compounds exhibited high incidence values across all populations (Table 1). The SIMPER analysis also revealed that of the ten most dissimilar compounds between U.S. and Mesoamerica, eight were present in both population sets (Table 1; Fig. 6 except panels $\mathrm{f}, \mathrm{j}$ ). Of these, six were present in more individuals and had greater relative quantities in Mesoamerican than in U.S. populations (Fig. 6a,c,d,e,g,h). The two remaining compounds (eugenol and caryophyllene) were present in more individuals and had greater relative amounts in U.S. than Mesoamerican populations (Fig. 6b,i). Triclopyr BEE and benzyl benzoate were present only in U.S. and Mesoamerica, respectively (Fig. 6f,j). Triclopyr BEE contributed up to $69 \%$ of total fragrance composition in U.S. bees, and it was detected in $63 \%$ of individuals $(N=$ 43). Benzyl benzoate contributed up to $17.84 \%$ of the fragrance composition in individual bees, and it was detected in $18 \%$ of Mesoamerican individuals $(N=71)$.

We also conducted a SIMPER analysis among all seven populations. In 15 of the 21 possible pairwise comparisons, five compounds jointly explained $>50 \%$ of the observed fragrance dissimilarity (Supplementary Material Appendix 3). In all but two pairwise comparisons, El Remate (Mexico) v. ACG (Costa Rica) and El Remate v. Monte Pio (Mexico), the compounds HNDB4 and eugenol ranked among the top five compounds with highest dissimilarity between populations (Supplementary Material Appendix 3). Sesquiterpene compounds, followed by monoterpenes, contributed the most to the observed dissimilarity between populations (Table 1; Online Appendix 3). Although diverse, triterpenes contributed little or nothing to population structuring.

As described earlier for E. aff. viridissima (Eltz et al., 2008), four stereoisomers of the compound HNDB were found in male perfumes (Supplementary Material Fig. 1). The compound HNDB4 was by far the most abundant stereoisomer in the majority of populations, except in El Remate (Mexico). The fragrances of all individuals in El Remate had greater relative amounts of the stereoisomers HNDB1, HNDB2, and HNDB3 than all other populations. In some cases, other stereoisomers were more abundant than HNDB4 itself (Supplmentary material Fig. 1). Because our spectral searches against published libraries did not return any significant matches for triclopyr BEE, the identification of this compound was conducted by comparison against analytical standards provided by Dow AgroSciences (Indianapolis, IN, US). Both the retention time and EI-spectrum of the analytical standard of triclopyr 2butoxyethyl ester (triclopyr BEE) perfectly matched peaks in our samples (Fig. 7). Male E. aff. viridissima were observed displaying fragrance collecting behavior on areas where the herbicide Garlon had been applied in Fern Forest (Pemberton, Pers. Obs.). Triclopyr BEE is one of three available active ingredients present in commercially available herbicides (including Garlon) used for broadleaf weed control. The other active ingredients are triclopyr acid and triclopyr triethylamine salt (TEA).

In our laboratory bioassay, we presented 20 male bees with three hexane leg extracts containing large amounts of triclopyr BEE and trace amounts of other volatile compounds. Within the first two minutes of exposure, six, five, and six individual bees exhibited obvious collecting behavior in the three trials, respectively. Controls (labial lipid extracts) did not elicit fragrance-collecting behavior in any of the trials.

Our bioassay using purified aliquots of triclopyr BEE with native bee populations in the field revealed that male $E$. aff. viridissima are attracted to, and collect, this volatile compound. A total of five male $E$. aff. viridissima approached the filter paper with triclopyr BEE, landed on it, and performed typical collection behavior. Other males circled the filter paper but did not land on it. 
Fig. 6 Boxplots of per-capita relative concentration (relative amount of each compound over all compounds) of ten chemicals detected in bees sampled from native (Mesoamerica) and naturalized (U.S.) populations. These ten compounds had the greatest contributions to dissimilarity between native and naturalized populations
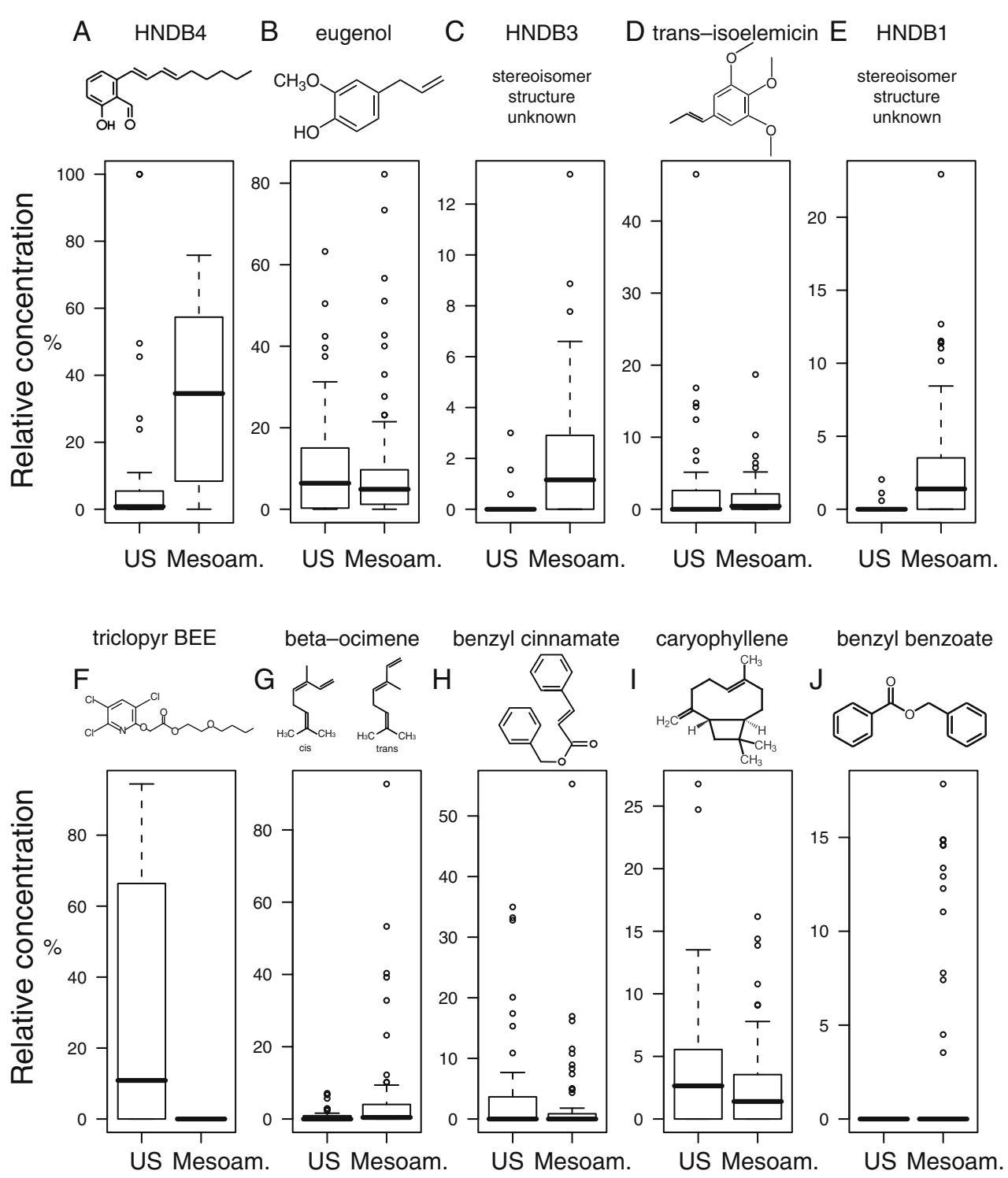

\section{Discussion}

Euglossine bees acquire species-specific fragrance bouquets from a great diversity of floral and non-floral sources (Williams, 1982; Whitten et al., 1993; Ramírez et al., 2002; Pemberton and Wheeler, 2006; Zimmermann et al., 2009a). As a result, individual perfume phenotypes may not be attributable to single sources, but instead, emerge from multiple visits to numerous hosts (Eltz et al., 2005a; Pemberton and Wheeler, 2006). Although sympatric species of orchid bees, and particularly closely related lineages (Eltz et al., 1999; Zimmermann et al., 2006, 2009a), exhibit divergent perfume phenotypes, it has been unclear to what extent fragrances vary across geographic regions within lineages. Our results indicate that male $E$. aff. viridissima maintain most of the individual compounds of their fragrance phenotypes across distant populations in disparate habitats, but a few major (abundant) compounds can be present or absent from perfume bouquets. Moreover, since populations of $E$. aff. viridissima in Florida have been stable for at least seven years, our data suggest that the observed phenotypic changes have negligible effects on population viability. This observation is congruent with the previous report that male Euglossa tridentata and E. erythrochlora sampled in Isla del Caño, an island $17 \mathrm{~km}$ off the coast of Costa Rica, collect substantially fewer compounds per capita than in mainland populations (Eltz et al., 2005a, Pers. Obs.).

The remarkable qualitative consistency we found in fragrance phenotypes is at odds with the considerable habitat variability among the seven sites sampled, but supports the hypothesis that orchid bee perfume preferences are under strong selection (Zimmermann et al., 2009a). The differences in perfume composition that we observed between native and naturalized populations could be 


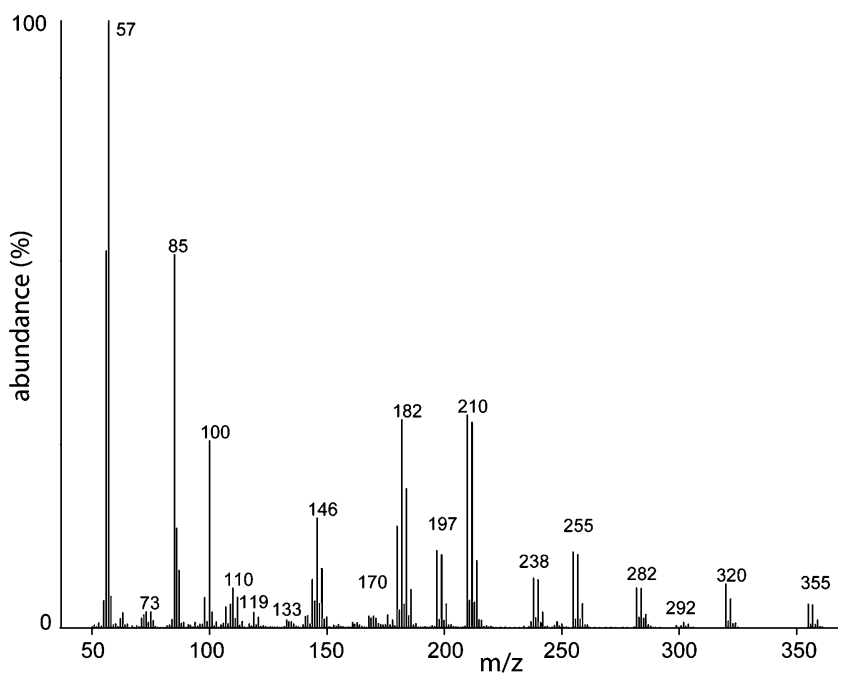

Fig. 7 Mass spectrum of triclopyr 2-butoxyethyl ester, a prevalent compound found in leg extracts of males from naturalized populations of Euglossa aff. viridissima in Florida (U.S.) and absent in native (Mesoamerican) populations

attributed to environmental factors, to differences in chemical preference between populations, or to both. Several factors may influence intraspecific fragrance variation. First, species turnover (beta diversity) of fragrance hosts across habitats may force phenotypic changes via chemical abundance and availability. In fact, a recent survey of volatile compounds present in resins of Amazonian trees revealed not only a vast diversity of monoterpenes and sesquiterpenes, but also pronounced chemical turnover across tree species from different habitats (Courtois et al., 2009). Second, strong female preference for certain fragrance phenotypes may restrict the amount of perfume phenotypic plasticity. Although direct evidence for female choice of fragrance phenotypes is still missing for orchid bees, perfume phenotypes are likely to be under strong sexual selection (Zimmermann et al., 2009a, b). Hence, it is also conceivable that, at evolutionary timescales, new male fragrance phenotypes may emerge through novel female preferences for perfumes that indicate mate genotypic quality or simply exploit a sensory bias (Andersson, 1994).

Genetic data indicate that naturalized populations of $E$. aff. viridissima have gone through a severe population bottleneck (Zimmermann et al. unpublished data), and therefore it is possible that population differences in perfume phenotypes have an underlying genetic basis. Rapid evolutionary change in pheromone phenotypes has been demonstrated in several species of insects (Lofstedt, 1993; Takanashi et al., 2005; Groot et al., 2009), and could be tested in orchid bees via common garden experiments.

The orchid bee lineage E. aff. viridissima is broadly sympatric with its sister taxon (E. viridissima) throughout most of Mesoamerica (Eltz et al. unpublished data). The main difference in the perfumes of these two lineages is the presence of HNDB in E. aff. viridissima and its complete absence in E. viridissima (Eltz et al., 2008, unpublished data). Our data corroborated the presence of HNDB as a major fragrance compound in both native and naturalized populations of $E$. aff. viridissima and, interestingly, showed that the four known structural stereoisomers of HNDB (Eltz et al., 2008) occur in similar ratios in all introduced and most native populations. Highly variable ratios of HNDB stereoisomers were found only in one population (El Remate, Mexico). Although the source of HNDB remains unknown, this either suggests that bees acquire HNDB from the same host in both native and introduced populations, but from a different source in El Remate, or that environmental differences between El Remate and the other sites induced changes in the production of HNDB stereoisomers by the same host. The chemical structure of HNDB is unique, but resembles compounds produced by phytopathological fungi (Suzuki et al., 1987: Berkaew et al., 2008).

The presence of large quantities of triclopyr BEE in leg extracts of naturalized bee populations, together with our field and laboratory bioassays, demonstrates that male bees are attracted to and actively collect this herbicide active ingredient. Indeed, previous studies have shown that males of the orchid bee Eufriesea purpurata from Brazil collect large quantities of the synthetic pesticide DDT (Roberts et al., 1982; Walter and Roberts, 2007). Although we did not detect triclopyr BEE in leg extracts from native populations, male bees from Mesoamerica clearly were attracted to this compound. This result suggests that a pre-existing sensory bias, rather than rapid adaptation among introduced populations, may explain the collection of organohalogen synthetic chemicals by male orchid bees. Both behavioral and neurophysiological approaches are needed to elucidate the sensory basis of this unusual behavior.

Our results illustrate that male $E$. aff. viridissima collect a broad diversity of molecules in native and introduced populations. As shown previously for orchid bees (e.g., Williams and Whitten, 1983; Eltz et al., 1999, 2005a; Zimmermann et al., 2009a), fragrance bouquets were composed mainly of monoterpenes, bicyclic sesquiterpenes, and aromates. We found that perfume compounds that differed the most in abundance among populations also tended to be common to all populations. This was the case for several monoterpene, sesquiterpene, and aromate compounds. For example, whereas eugenol and HNDB were present in $>73 \%$ of the sampled individuals and contributed significantly to population dissimilarity, most rare compounds contributed little to population dissimilarity. We confirmed this pattern by plotting the contribution to the 
dissimilarity between U.S. and Mesoamerica as a function of compound average abundance (data not shown).

Despite contributing little to overall population differences, rare compounds were diverse in our dataset. For instance, we detected numerous triterpenes in fragrances of E. aff. viridissima, but most of them were present in few individuals and in low concentrations. Because triterpenes exhibit high molecular weights $(>400)$, and thus may not volatilize at ambient temperature, they may constitute poor airborne signaling molecules. We speculate that orchid bees actually collect triterpenes accidentally since they are common components of tree resins (e.g., Burseraceae), which are known to contain numerous volatile compounds (De La Cruz-Cañizares et al., 2005; Courtois et al., 2009). Hence, most of the compounds that were abundant in our dataset, such as sesquiterpenes, monoterpenes, and aromates, contributed the most to population differences, and also are likely to constitute the main courtship signaling molecules in orchid bees.

In summary, the preference for most fragrance compounds acquired by orchid bees appears to be under strong selection. Evidence for this comes from the fact that male bees managed to collect similar compounds across disparate habitats ranging from tropical rain forests to tropical dry forests, to mangroves, to disturbed habitats. However, we also found evidence for pronounced quantitative and qualitative changes across geographic (and possibly genetic) gradients. Previous studies have suggested that saltational changes in fragrance preferences may constitute an important mechanism of evolution of perfume phenotypes as well as speciation in orchid bees (Eltz et al., 2008; Zimmermann et al., 2009a). Because mutations that affect the olfactory system of orchid bees can induce the acquisition or loss of specific chemicals, perfume phenotypes have the potential to evolve fast enough to facilitate lineage differentiation (Eltz et al., 2008; Jackson, 2008; Zimmermann et al., 2009a). Our analysis highlights the opportunistic capacity that orchid bees have to incorporate novel compounds in their perfumes, as illustrated by the gain and loss of several compounds in naturalized populations. Whether population-level changes in perfume phenotypes have underlying genetic components, and are thus subject to selection, should be a fruitful area of future research.

Acknowledgements We thank Rachel Taylor and Anthony Boughton for assistance during fieldwork in Florida. We thank the staff members from the USDA-Agricultural Research Service's Invasive Plant Research Laboratory for providing assistance and allowing access to facilities, and Javier Quezada-Euan and the staff members of the Department of Apiculture at UADY, Mexico, for their long-term cooperation. We thank Roger Blanco and Maria Chavarría for help with collection permits in the Area de Conservación Guanacaste (Costa Rica). Elena Ormeño kindly provided the internal standards used in compound verification; Dong-Hwan Choe provided assistance in the identification of triclopyr BEE. We thank Jenny Jacobs for comments on an earlier version of the manuscript. This research was partly supported by USDA grant NRI-CGP-2008-3530204680 to NDT.

Open Access This article is distributed under the terms of the Creative Commons Attribution Noncommercial License which permits any noncommercial use, distribution, and reproduction in any medium, provided the original author(s) and source are credited.

\section{References}

ADAMS, R. P. 2001. Identification of Essential Oil Components by Gas Chromatography / Quadrupole Mass Spectroscopy. Allured Publishing Corporation, Carol Stream, IL, 804 pp.

Andersson, M. 1994. Sexual Selection. Princeton University Press, Princeton, NJ, $624 \mathrm{pp}$.

BEMBÉ, B. 2004. Functional morphology in male euglossine bees and their ability to spray fragrances (Hymenoptera, Apidae, Euglossini). Apidologie 35:283-291.

Berkaew, P., Soonthornchareonnon, N., SAlasawadee, K., ChanthaKet, R., and IsAKA, M. 2008. Aurocitrin and related metabolites from the wood-decay fungus Hypocrea sp. J. Nat. Prod. 71:902-904.

Billeter, J. C., Atallah, J., Krupp, J. J., Millar, J. G., and LEVINE, J. D. 2009. Specialized cells tag sexual and species identity in Drosophila melanogaster. Nature 461:987-991.

Cardé, R. T., Cardé, A. M., Hill, A. S., and Roelofs, W. L. 1977. Sex-pheromone specificity as a reproductive isolating mechanism among sibling species Archips argyrospilus and A. mortuanus and other sympatric Tortricine moths (Lepidoptera: Tortricidae). J. Chem. Ecol. 3:71-84.

Clarke, K., and Gorley, R. 2006. PRIMER v6: User Manual/ Tutorial. PRIMER-E, Plymouth.

Courtois, E. A., Paine, C. E. T., Blandinieres, P. A., Stien, D., Bessiere, J. M., Houel, E., Baraloto, C., and Chave, J. 2009. Diversity of the volatile organic compounds emitted by 55 species of tropical trees: a survey in French Guiana. J. Chem. Ecol. 35:1349-1362.

DARWIN, C. 1862. On the Various Contrivances by which British and Foreign Orchids are Fertilised. John Murray, London.

De la Cruz-Cañizares, J., Domenech-Carbo, M. T., GimenoAdelantado, J. V., Mateo-Castro, R., and Bosch-Reig, F. 2005. Study of Burseraceae resins used in binding media and varnishes from artworks by gas chromatography-mass spectrometry and pyrolysis-gas chromatography-mass spectrometry. J. Chrom. A 1093:177-194.

Dodson, C. H., Dressler, R. L., Hills, H. G., Adams, R. M., and WiLLIAMS, N. H. 1969. Biologically active compounds in orchid fragrances. Science 164:1243-1249.

DRESSLER, R. L. 1968. Pollination by euglossine bees. Evolution 22:202-210.

DRESSLER, R. L. 1982. Biology of the orchid bees (Euglossini). Annu. Rev. Ecol. Syst. 13:373-394.

Eltz, T., Whitten, W. M., Roubik, D. W., and Linsenmair, K. E. 1999. Fragrance collection, storage, and accumulation by individual male orchid bees. J. Chem. Ecol. 25:157-176.

Eltz, T., RoubiK, D. W., and Whitten, M. W. 2003. Fragrances, male display and mating behaviour of Euglossa hemichlora: a flight cage experiment. Physiol. Entomol. 28:251-260.

Eltz, T., RoubiK, D. W., and LunAu, K. 2005a. Experiencedependent choices ensure species-specific fragrance accumulation in male orchid bees. Behav. Ecol. Sociobiol. 59:149-146. 
Eltz, T, SAger, A, and LunAu, K. 2005b. Juggling with volatiles: exposure of perfumes by displaying male orchid bees. J. Comp. Physiol. A 191:575-581.

Eltz, T., Zimmermann, Y., Haftmann, J., Twele, R., Francke, W., QUEZADA-EUAN, J. J. G., and LUNAU, K. 2007. Enfleurage, lipid recycling and the origin of perfume collection in orchid bees. Proc. Roy. Soc. B-Biol. Sci. 274:2843-2848.

Eltz, T., Zimmermann, Y., Pfeiffer, C., Pech, J. R., Twele, R., Francke, W., QueZadA-EuAn, J. J. G., and LunAU, K. 2008. An olfactory shift is associated with male perfume differentiation and species divergence in orchid bees. Curr. Biol. 18:1844-1848.

Groot, A. T., Inglis, O., Bowdridge, S., Santangelo, R. G., Blanco, C., Lopez, J. D., Vargas, A. T., Gould, F., and SCHAL, C. 2009. Geographic and temporal variation in moth chemical communication. Evolution 63:1987-2003.

Hinojosa-Diaz, I. A., Feria-Arroyo, T. P., and Engel, M. S. 2009. Potential distribution of orchid bees outside their native range: The cases of Eulaema polychroma (Mocsary) and Euglossa viridissima Friese in the USA (Hymenoptera: Apidae). Divers. Distrib. 15:421-428.

JACKSON, D. E. 2008. Sympatric speciation: Perfume preferences of orchid bee lineages. Curr. Biol. 18:R1092-R1093.

LiU, H., and Pemberton, R. W. 2009. Solitary invasive orchid bee outperforms co-occurring native bees to promote fruit set of an invasive Solanum. Oecologia 159:515-525.

LOFSTEDT, C. 1993. Moth pheromone genetics and evolution. Philos. T. Roy. Soc. B 340:167-177.

NoJima, S., Apperson C. S., and Schal, C. 2008. A simple, convenient, and efficient preparative GC system that uses a short megabore capillary column as a trap. J. Chem. Ecol. 34:418-428.

Pemberton, R. W., and LiU, H. 2008. Naturalized orchid bee pollinates resin-reward flowers in Florida: novel and known mutualisms. Biotropica 40:714-718.

Pemberton, R. W., and Wheeler, G. S. 2006. Orchid bees don't need orchids: Evidence from the naturalization of an orchid bee in Florida. Ecology 87:1995-2001.

RAMíREZ, S. R. 2009. Quick guide: Orchid bees. Curr. Biol. 19:10611063.

RAmíreZ, S., DreSSler, R. L., and OSPINA, M. 2002. Orchid bees (Hymenoptera: Apidae: Euglossini) from the Neotropical Region: A species checklist with notes on their biology. Biot. Colomb. 3:7-118.

Ramírez, S. R., RoubiK, D. W., Skov, C., and Pierce, N. E. 2010. Phylogeny, diversification patterns and historical biogeography of euglossine orchid bees (Hymenoptera: Apidae). Biol. J. Linn. Soc. 100:552-572. doi:10.1111/j.1095-8312.2010.01440.x

Roberts, D. R., Alecrim, W. D., Heller, J. M., Erhardt, S. R., and LimA, J. B. 1982. Male Eufriesia purpurata, a DDTcollecting bee in Brazil. Nature 297:62-63.

Roelofs, W. L., LiU, W., HaO, G., JiaO, H., Rooney, A. P., and LINN, C. E. JR. 2002. Evolution of moth sex pheromones via ancestral genes. Proc. Natl. Acad. Sci. USA 99:13621-13626.
RoubiK, D. W., and Hanson, P. E. 2004. Orchid Bees of Tropical America: Biology and Field Guide. INBIO, Heredia, Costa Rica.

SKOV, C., and WILEY, J. 2005. Establishment of the neotropical orchid bee Euglossa viridissima (Hymenoptera: Apidae) in Florida. Fla. Entomol. 88:225-227.

StennetT, M. D., and Etges, W. J. 1997. Premating isolation is determined by larval rearing substrates in cactophilic Drosophila mojavensis. III. Epicuticular hydrocarbon variation is determined by use of different host plants in Drosophila mojavensis and Drosophila arizonae. J. Chem. Ecol. 23:2803-2824.

Suzuki, M., Sugiyama, T., Watanabe, M., Murayama, T., and YAMASHITA, K. 1987. Synthesis and absolute configuration of pyriculol. Agric. Biol. Chem. 51:1121-1127.

SYMONDS, M. R. E., and Elgar, M. A. 2004. The mode of pheromone evolution: evidence from bark beetles. Proc. Roy. Soc. B-Biol. Sci. 271:839-846.

Symonds, M. R. E., and Elgar, M. A. 2008. The evolution of pheromone diversity. Trends Ecol. Evol. 23:221-228.

Takanashi, T., Huang, Y. P., Takahasi, K. R., Hoshizaki, S., TATSUKI, S., and ISHIKAWA, Y. 2005. Genetic analysis and population survey of sex pheromone variation in the adzuki bean borer moth, Ostrinia scapulalis. Biol. J. Linn. Soc. 84:143-160.

WALTER, V., and RoBERTS, D. 2007. Revisiting the organohalogens associated with 1979-samples of Brazilian bees (Eufriesea purpurata). Sci. Total Environ. 377:371-377.

WhitTen, W. M., Young, A. M., and Williams, N. H. 1989 Function of glandular secretions in fragrance collection by male euglossine bees (Apidae: Euglossini). J. Chem. Ecol. 15:12851295.

Whitten, W. M., Young, A. M., and Stern, D. L. 1993. Nonfloral sources of chemicals that attract male euglossine bees (Apidae: Euglossini). J. Chem. Ecol. 19:3017-3027.

WiLLIAMS, N. H. 1982. The biology of orchids and euglossine bees, pp. 119-171, in J. Arditti (ed.). Orchid Biology: Reviews and Perspectives, II. Cornell University Press, Ithaca, NY.

WILLIAMS, N. H., and WhitTEN, W. M. 1983. Orchid floral fragrances and male Euglossine bees: Methods and advances in the last sesquidecade. Biol. Bull. 164:355-395.

WyatT, T. D. 2003. Pheromones and Animal Behaviour: Communication by Smell and Taste. Cambridge University Press, Cambridge, $408 \mathrm{pp}$.

ZimmermanN, Y., RoubiK, D. W., and Eltz, T. 2006. Speciesspecific attraction to pheromonal analogues in orchid bees. Behav. Ecol. Sociobiol. 60:833-843.

ZimmermanN, Y., RamíreZ, S. R., and Eltz, T. 2009a. Chemical niche differentiation among sympatric species of orchid bees. Ecology 90:2994-3008.

Zimmermann, Y., RoubiK, D. W., QueZada-Euan, J. J. G., PAXtON, R. J., and Eltz, T. 2009b. Single mating in orchid bees (Euglossa, Apinae): implications for mate choice and social evolution. Insect. Soc. 56:241-249. 